VOL. $4(1971), 9-15$.

\title{
On the definition of saturated formations of groups
}

\section{John Cossey and Sheila Oates Macdonald}

We exhibit a closure operation which serves to define saturated formations of finite soluble groups.

\section{Introduction}

Classes of groups defined in terms of closure operations have proved both interesting and useful. Some classes originally defined in terms of more than one operation can be defined in terms of a single closure operation which is a product of the original operations, for example, varieties and formations. In this paper we attempt to do the same for saturated formations, with partial success. In Theorem 3.1 we obtain a closure operation for saturated formations of soluble groups. This particular operation is unusual in that it uses twice each of the original closure operations. We do not know if this is best possible, although it is not difficult to check that it is not possible to define such an operation using each of the original operations once only. Our techniques rely heavily on the solubility of the groups involved; we are unable to say anything in the insoluble case.

\section{Notation and preliminary results}

For notation and basic facts about formations we refer the reader to [2] or [3]. For the remainder of this paper all groups considered will be finite and soluble.

If $X$ is a class of groups, $f X$ will denote the formation and $s f X$

Received 11 August 1970. 
the saturated formation generated by $X$. If $X=\{G\}$ we will write $f G$ and $s f G$.

If $F$ is a formation, $G^{F}$ will denote the F-residual of $G$, that is,

$$
G^{F}=\cap\{N: N \diamond G \text { and } G / N \in F\} .
$$

If $F$ and $G$ are formations, then

$$
F G=\left\{G: G^{G} \in F\right\}
$$

is also a formation.

$S_{p}$ denotes the formation of $p$-groups, $p$ a prime.

A closure operation is a map $P$ defined on classes of groups which satisfies:-

(i) $X \leq Y \Rightarrow P X \leq P Y$;

(ii) $X \leq P X=P(P X)$.

We have:-

LEMMA 2.1. Q, $R_{0}$, and $\Phi$ are closure operations where:-

$$
\begin{aligned}
Q X= & \{H: G / N \simeq H \text { and } G \in X\} ; \\
R_{0} X= & \left\{H: N_{i} \triangleleft H, i=I, \ldots, n ; \bigcap_{i=1}^{n} N_{i}=I \text { and } H / N_{i} \in X\right\} ; \\
\Phi X= & \{H: H / \Phi(H) \simeq G / \Phi(G) \text { and } G \in X\} \text { where } \Phi(H) \text { is the Frattini } \\
& \text { subgroup of } H .
\end{aligned}
$$

We shall need the following well known fact (see, for example, the introduction to [1]).

LEMMA 2.2. $R_{0} Q X \leq Q R_{0} X=f X$.

The socle of a group $G$ is denoted by $\sigma G$. If $G$ has a unique minimal normal subgroup we call $G$ monolithic and $\sigma G$ its monolith.

We say that a group with trivial Frattini subgroup is $\Phi$-free. We need:- 
LEMMA 2.3. If $F$ is a saturated formation and $x$ the class of Ф-free monolithic groups in $F$, then

$$
F=\Phi R_{0} X \text {. }
$$

Proof. Let $G \in F$, then $H=G / \Phi(G)$ is $\Phi$-free. Let $\sigma H=N_{1} \times \ldots \times N_{r}$ be the decomposition of the socle of $H$ into minimal normal subgroups of $H$; then $H \in R_{0}\left\{H / K_{i}, i=1, \ldots, r\right\}$ where $K_{i}$ is a maximal normal subgroup of $H$ containing $N_{1} \times \ldots \times N_{i-1} \times N_{i+1} \times \ldots \times N_{p}$ and avoiding $N_{i} \cdot$ Clearly $H / K_{i} \in F$ and is monolithic (with monolith isomorphic to $N_{i}$ ). But it is also Ф-free since it splits over its monolith.

LEMMA 2.4. Let $D$ be the set of $\Phi$-free monolithic quotients of the group $G$ and let $F_{p}$ be the formation generated by $\{D / \sigma D: D \in D$ and $p|| \sigma D \mid\}$ for each prime $p$ dividing $|G|$. Then if $H$ is a monolithic group in $s f G$ whose monolith is a p-group, $H \in S_{p} F_{p}$.

$$
\text { Proof. Let } F=p_{p}|| G \mid S_{p} F_{p} \text {. Clearly } s f G={ }_{n}^{U}\left(\Phi Q R_{0}\right)^{n_{F}} \text {. Let } \quad H
$$

be a monolithic group in $\left(\Phi Q R_{0}\right)^{n_{F}}$ whose monolith is a p-group. We prove by induction on $n$ that $H \in S_{p} F_{p}$. For $n=0$, we have $H \in F$ and so either $H \in S_{p} F_{p}$ or $H \in F_{q}$ for some $q \neq p$. In the latter case

$$
H \in Q R_{\mathrm{o}}\{D / \sigma D: q|| \sigma D \mid\}
$$

As in [4] we can obtain an expression for $H$ as a quotient of a subdirect product of monolithic groups whose monoliths are p-groups. It follows that $H / \sigma H \in F_{p}$ and so $H \in S_{p} F_{p}$.

Now take $n \geq 1$ and assume the lemma true for $n-1$. Since $\sigma H$ is a p-group so also is $\sigma(H / \Phi(H))$. But $H / \Phi(H) \in Q R_{0}\left(\Phi Q R_{0}\right)^{n-1} F$. Again, using the methods of [4] we obtain that $H / \Phi(H) \in Q R_{0}\left\{A_{i}\right\}$ where the $A_{i}$ are monolithic with $\sigma A_{i}$ a $p$-group and belong to $\left(\Phi Q R_{0}\right)^{n-1} F$. By the induction hypothesis $A_{i}$ and hence $H / \Phi(H)$ belongs to $S_{p} F_{p}$. Thus 
$H \in S_{p} F_{p}$ as required.

The next lemma is rather technical in nature, and may be regarded as a generalisation of [3] VI 7.22. For notation and results about varieties of groups we refer the reader to [6].

LEMMA 2.5. Let $M$ be a faithful $\mathrm{GF}(p) G$ module of rank $r$. Then there exists a group $L$ which is the split extension by $G$ of a metabelian class $c$ p-group $N$ such that $\gamma_{c}(N) \simeq M^{\otimes c}$, where $\gamma_{c}(N)$ is regarded as a $\mathrm{GF}(p) G$-module, and $M^{\otimes c}$ denotes the c-fold tensor power of $M$.

Proof. Let $F$ be the free group of rank $r c$ in the variety consisting of the groups of class $c$ in the product variety $\stackrel{A}{A} \stackrel{A}{p}$, that is,

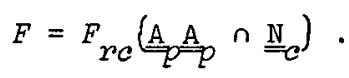

Suppose $F=\left\langle x_{i j}: 1 \leq i \leq c, 1 \leq j \leq r\right\rangle$; then for each $g \in G$, we can define an action on the generators of $F$ by letting $g$ act on the sets

$$
T_{i}=\left\{x_{i j}: 1 \leq j \leq r\right\}
$$

as it does on a set of generators for $M$, and extending this action to an automorphism of $F$. We can thus regard $G$ as a set of automorphisms of $F$. Now let $K$ be the subgroup of $F$ generated by all those left-normed commutators which have an entry from some $T_{i}$ in more than one place or which are of weight $c$ and have an entry in the first two places other than from $T_{1}$ and $T_{2}$. Let $J$ be the subgroup of $F$ generated by those left-normed commutators of weight $c$ of the form:-

$$
\left[x_{1 j_{1}}, \ldots, x_{c j_{c}}\right] \text {. }
$$

Then it can be extracted from 4.05 of [5] that $K \cap J=1$. Also it is readily checked that $K$ and $J$ are normal in $F$ and are mapped to themselves by the automorphisms induced by $G$. Consider $F / K$. $G$ can be considered as a subset of the automorphism group of $F / K$, and it is easy to check that it is actually a subgroup. We see that $N=F / K$ satisfies 
the required conditions; for $\gamma_{c}(N)$ is an elementary abelian p-group of rank re and the mapping

$$
\psi: M^{\otimes c}+\gamma_{c}(N)
$$

defined by

$$
x_{1 j_{1}} \otimes \ldots \otimes x_{c j_{c}} \psi=\left[x_{1 j_{c}}, \ldots, x_{c j_{c}}\right]
$$

is clearly a module isomorphism. Hence we may take $L$ to be the split extension of $N$ by $\boldsymbol{G}$.

\section{Proof of the theorem}

THEOREM 3.1. $s f X=\left(\Phi Q R_{0}\right)^{2} X$.

Proof. We consider first the case in which $X=\{G\}$. By Lemma 2.3 $s f G=\Phi R_{0} y$ where $y$ is the set of $\Phi$-free monolithic groups in $s f G$. Since $R_{0} Q R_{0}=Q R_{0}$, the proof will be complete if we show that

$$
y \leq Q R_{0} \Phi Q R_{0} G
$$

and, by Lemma 2.4, it will suffice to prove that if $B$ is a $\Phi$-free monolithic group in $S_{p} F$ then

$$
H \in Q R_{0} \Phi Q R_{0} G \text {. }
$$

Since

$$
F_{p}=Q R_{0}\{D / \sigma D\} \leq Q R_{0} G
$$

we can assume $H \notin F_{p}$; and so $\sigma H$ is a p-group. Since $H$ is $\Phi$-free this is also its Fitting subgroup, and so $H / \sigma H \in F_{p}$; say $H / \sigma H \simeq S / T$ where $S$ is subdirect in $\prod_{i=1}^{s} A_{i}, A_{i} \simeq D_{i} / \sigma D_{i}$. Then $B=\sigma H$ is an irreducible $\mathrm{GF}(p) S / T$ module, and a fortiori, an irreducible $S$ module. There is a natural epimorphism from $\prod D_{i}$ to $\prod A_{i}$. Let $\tilde{S}$ be the inverse image in $\prod D_{i}$ of $S$. Then, by the definition of the $D_{i}, \tilde{S}$ 
is the split extension by $S$ of a faithful $\mathrm{GF}(p) S$ module $M$, and $\tilde{S} \leq Q R_{0} G$. By [3] VI 7.19, $B$ is a composition factor of $M^{\otimes C}$ for some c. Consider the group $L$ defined as in Lemma 2.5. Then

$$
L / \Phi(L) \in R_{0} \tilde{S} \leq Q R_{0} G
$$

and so $L \in \Phi Q R_{0} G$. By [3] VI 7.21 the split extension of $B$ by $S$ lies in $f L$ and hence so does B.S/T. But this is $H$. Hence

$$
H \in Q R_{0} L \leq Q R_{0} \Phi Q R_{0} G
$$

as required.

For the general case consider $K \in s f X$. Then $K$ is obtained from $X$ by a finite number of applications of $\Phi, Q$ and $R_{0}$, say

$K \in\left(\Phi Q R_{0}\right)^{n} X$. We prove by induction on $n$ that $K$ is obtained from a finite number of elements of $X$; the result being true for $n=0$. Now

$$
K \in \Phi Q R_{0}\left(\Phi Q R_{0}\right)^{n-1} \chi,
$$

that is, $K / \Phi(K) \simeq S / T$ where $S \leq X_{1} \times \ldots \times X_{\gamma_{0}}$ and $X_{i} \in\left(\Phi Q R_{0}\right)^{n-1} X$. By the induction hypothesis, each $X_{i}$ arises from only finitely many members of $X$ and hence so also does $K$. Thus $K \in s f G$ where $G$ is the direct product of these members of $X$ and hence

$$
K \in\left(\Phi Q R_{0}\right)^{2} G \leq\left(\Phi Q R_{0}\right)^{2} X
$$

\section{References}

[1] R.M. Bryant, R.A. Bryce and B. Hartley, "The formation generated by a finite group", Buz2. Austral. Math. Soc. 2 (1970), 347-357.

[2] Wolfgang Gaschütz, Selected topics in the theory of soluble groups (Lectures given at the Ninth Summer Research Institute of the Australian Mathematical Society in Canberra, 1969. Notes by J. Looker). 
[3] Bertram Huppert, Endliche Gmopen I (Die Grundlehren der mathematischen Wissenschaften, Band 134, Springer-Verlag, Berlin, Heidelberg, New York, 1967).

[4] L.G. Kovács and M.F. Newman, "On critical groups", J. AustraZ. Math. Soc. 6 (1966), 237-250.

[5] L.G. Kovács and M.F. Newman, "On non-Cross varieties of groups", $J$. Austral. Math. Soc. (to appear).

[6] Hanna Neumann, Varieties of groups (Ergebnisse der Mathematik und ihrer Grenzgebiete, Band 37, Springer-Verlag, Berlin, Heidelberg, New York, 1967).

School of General Studies,

The Australian National University,

Canberra, ACT,

and

The University of Queensland,

St Lucia, Queensland. 